\title{
EDITORIAL
}

\section{The benefits of speaking a common language}

This issue marks the publication of a Consensus Statement that proposes a classification system

for the evolutionary and ecological features of cancers.

All too often, getting a group of scientists to agree on something presents a challenge. However, in many cases agreement is crucial to propel a field of research forward. Agreement, or consensus, does not necessarily mean the answers are all known and the results are final. Rather, consensus can be a jumping-off point for further refinement of a system, ensuring that researchers in that field are using a common language in their data collection and publication. The goal of such consensus is to make studies by different groups of researchers more comparable, hopefully accelerating advancement in the field.

In this issue, Nature Reviews Cancer are publishing our first Consensus Statement article, which proposes the framework for a classification system for the evolutionary and ecological features of cancers ${ }^{1}$. Written by Carlo Maley and 21 fellow experts in the fields of cancer evolution and cancer ecology, this article arose from a consensus conference at the 2016 Wellcome Trust Evolution and Ecology of Cancer summer school.

The authors have developed a framework that includes both an evolutionary index (Evo-index), which describes the evolvability of a population of tumour cells, and an ecological index (Eco-index), which describes potential selective pressures imposed on tumour cells by the surrounding microenvironment. Each of these indices in turn includes two measurements. The Evo-index includes measurements of the diversity of neoplastic cells (intratumoural heterogeneity), as well as parameters that describe how that diversity changes over time. The Eco-index includes measurements that indicate various hazards to tumour cell survival and that specify the resources available to tumour cells.

In total, this leads to 16 different classes of tumour, although it is possible that not all 16 will be important clinically. Overall, the aim is for such measurements to identify different categories of tumour with different prognoses, and to determine how each tumour category will respond to interventions as well as how each would best be managed clinically. For example, some tumours are likely to have few resources along with little diversity or capacity to evolve, but have high levels of immune predation (hazards) that would likely signify a good patient prognosis. Those patients with the worst prognosis are likely to be those whose tumours have high genetic diversity and are poised to evolve rapidly, with plenty of resources but low levels of immune predation or other hazards.

Although the Evo- and Eco-indices at this stage are largely based on theory regarding evolutionary and ecological dynamics, and the authors have not recommended specific assays that must be used to measure each parameter, the hope is that this consensus framework will lead to methodological and technical innovations to quantify these key components of tumour evolution and ecology. The authors argue that from a clinical perspective, our understanding of the evolution and ecology of cancer is similar to that of psychiatry, specifically mental illnesses, in the 19th century. At that time, there was no standard classification system for such illnesses, which made it difficult for those treating these patients to make progress. Classification systems were published in 1933 (REF. 2), and then in 1952 (REF. 3). Although these systems had flaws, they enabled those clinician scientists treating these patients and researching these illnesses to speak to each other in a common language; this then facilitated refinement of the classifications and improvements in treatment.

We would encourage those readers who work with cancer patients and clinical samples to begin applying this consensus framework in their studies, collecting data on the four components of the Evo- and Eco-indices. This should help us to better understand the best measurements to use for each component, the utility of these indices for patient prognosis and how the different tumour types respond to different types of intervention. This will also allow the indices to be appropriately tested and refined so that we can move towards a time where we are all speaking the same language when it comes to measures of the evolution and ecology of cancer; ultimately it is hoped that this will directly improve the management and treatment of patients.

\footnotetext{
Maley, C. C. et al. Nat. Rev. Cancer http://dx.doi.org/10.1038/ nrc. 2017.69 (2017)

Logie, H. B. Can. Med. Assoc. J. 29, 193-194 (1933).

3. American Psychiatric Association. Diagnostic and Statistical Manual of Mental Disorders (American Psychiatric Association, 1952).
} 